\title{
Des femmes en gris
}

\section{Liliane Héroux}

Volume 36, 1969

URI : https://id.erudit.org/iderudit/1007294ar

DOI : https://doi.org/10.7202/1007294ar

Aller au sommaire du numéro

Éditeur(s)

Les Éditions Historia Ecclesiæ Catholicæ Canadensis Inc.

ISSN

0318-6172 (imprimé)

1927-7067 (numérique)

Découvrir la revue

Citer cet article

Héroux, L. (1969). Des femmes en gris. Sessions d'étude - Société canadienne d'histoire de l'Église catholique, 36, 49-56. https://doi.org/10.7202/1007294ar

Tous droits réservés @ Les Éditions Historia Ecclesiæ Catholicæ Canadensis Inc., 1970
Ce document est protégé par la loi sur le droit d'auteur. L'utilisation des services d'Érudit (y compris la reproduction) est assujettie à sa politique d'utilisation que vous pouvez consulter en ligne.

https://apropos.erudit.org/fr/usagers/politique-dutilisation/ 


\section{Des femmes en gris}

Le 25 août 1849, les deux quotidiens de Québec, Le Canadien et Le Journal de Québec, annonçaient comme un événement d'importance, l'arrivée dans la ville de nouvelles religieuses, venues de Montréal. Lisons ce communiqué :

Six Sœurs de la Charité de l'Hôpital Général de Montréal sont arrivées en cette ville pour y fonder un établissement du même genre. Elles doivent se livrer à l'instruction des jeunes filles pauvres, prendre soin des orphelins et soigner les malades à domicile. C'est dans l'asile des orphelins, dirigé par la Société des Dames charitables de Québec, que ces saintes filles vont commencer à exercer leur zèle. Plus tard elles ajouteront aux œuvres que nous venons de citer, celles de prendre soin des infirmes et d'élever les enfants abandonnés. Les citoyens de cette ville doivent s'estimer heureux de voir au milieu de nous ces Filles de Saint-Vincent-de-Paul, dont le zèle et la charité ont embrassé le monde entier.

Ce reportage de l'époque manquait un peu d'objectivité : «C'est dans l'asile des orphelins que ces saintes filles..." C'est une canonisation populaire et un peu hâtive; mais elle s'explique. Québec connaissait les Ursulines depuis Marie de l'Incarnation, les Hospitalières avec Catherine de Saint-Augustin, la Congrégation de Notre-Dame et sa Mère Bourgeoys. Et puis, bien que les Seurs Grises aient paru dans l'histoire cent ans plus tard, on avait entendu parler de Mère d'Youville et de son œuvre à Montréal, c'était l'éloge d'une sainte qui était parvenu à Québec. De là, pour l'opinion, à conclure que les nouvelles venues pouvaient bien être des saintes... Et quant à publier qu'elles étaient des Filles de Saint-Vincent-de-Paul, on peut encore excuser l'erreur de l'information. Car effectivement, l'archevêque avait eu l'intention de demander les Sœurs de Saint-Vincent-de-Paul de Paris, qu'on appelait Sours de la Charité; par la suite, il en avait été déconseillé devant certains inconvénients que présentait le projet. Mais, paix aux journalistes! Ces femmes en gris, qui sont-elles et d'où viennent-elles?

Avant de répondre, je dois m'excuser auprès des fervents de la vérité esthétique, d'appeler gris cette vague couleur qui, avec les années, a bel et bien glissé au beige. A la vérité, le gris est historique : c'est dès les premiers pas de son itinéraire spirituel que Marguerite d'Youville rencontra les contradictions humaines et l'odieuse calomnie; elle et ses compagnes furent accusées de fabriquer de l'eau-de-vie qu'on vendait aux sauvages et de s'en griser. Et c'est justement, de sa part, une réplique d'humilité qui lui a fait adopter la couleur de l'uniforme.

\section{DEUX PORTRAITS DE FEMMES}

Deux portraits de femmes s'offrent à nos yeux lorsque nous ouvrons l'histoire des Sœurs de la Charité à Québec : Marguerite d'You- 
ville et Anne-Marcelle Mallet. Ces figures de Canadiennes doivent être remises en mémoire et rafraîchies; mais pour toutes les deux, il faut dire qu'elles étaient plutôt effacées, comme la discrétion de leurs vertus et le gris de leur robe.

Marie Marguerite Du Frost de la Jemmerais, naquit à Varennes en 1701, l'année même où mourut Marguerite Bourgeoys. Elle vécut 70 ans de ce siècle où l'Église canadienne, rudement éprouvée, chancelait sur ses bases : le pays était pauvre et la guerre grondait à ses portes. Sur ia place de l'église, à son village natal, un monument est élevé à sa gloire, depuis que Rome l'a mise au rang des Bienheureux. Sur le socle, ces mots : Elle aima beaucoup Jésus-Christ et les pauvres. Voilà en résumé la vie de Mère d'Youville, première fondatrice canadienne. Cette femme a une histoire qui a tenté la plume de plusieurs écrivains, à diverses époques. L'un de ses fils d'abord, l'abbé Charles-Madeleine Du Frost, qui fut curé à Boucherville; il a laissé des cahiers manuscrits, une biographie de sa mère et des lettres; deux Sulpiciens : Messieurs Sattin et Faillon, 1852; Madame Berthe Jetté, 1900; Sœur Fauteux, s.g.m., 1929; Sœur Sainte-Blanche, s.c.q., 1932 : Une disciple de la croix; Madame Albertine Ferland-Angers : Mère d'Youville, première fondatrice canadienne, 1945; Sœur Estelle Mitchell, Elle a beaucoup aimé, 1959, et d'autres. A l'occasion de la béatification, il y a 10 ans, sur tout le réseau de la radio d'État, on avait longuement parlé de Mère d'Youville, dans une série d'émissions: Les visages de lamour, et, s'il faut être complet, dans un téléthéâtre, dont il vaut mieux ne pas évoquer avec trop d'insistance le souvenir décevant.

Au vieux manoir de Varennes, Marguerite ne connut que quelques années de joie; elle n'avait que sept ans quand mourut son père, Christophe Du Frost de la Jemmerais, gentilhomme de la vieille noblesse bretonne, capitaine de l'armée en Nouvelle-France, venu au pays en 1687. Sa mère, Marie-Renée Gaultier de Varennes, était fille de Gaultier de Varennes, gouverneur de Trois-Rivières et petite-fille de Pierre Boucher de Boucherville, ancien gouverneur de la même place. La famille en deuil fut vite dépourvue et vouée aux privations et à la gêne. L'enfant, aînée de cinq frères et sœurs, devint précocement sérieuse. A 12 ans, grâce à des secours fournis surtout par son aïeul, le vieux seigneur de Boucherville, elle fut admise au pensionnat des Ursulines de Québec, où passait l'élite féminine de la colonie et où Marguerite fit sa première communion, dans la chapelle de ce Monastère où nous nous trouvons présentement.

Revenue au foyer, elle partage avec sa mère les soins de la maison et doit fournir un supplément de travail pour gagner la vie des siens. A 20 ans, cette jeune fille a la beauté, le charme, l'élégance et une très suffisante instruction; elle fut beaucoup remarquée dans la société 
distinguée de Ville-Marie et elle agréa les hommages d'un jeune prétendant, héritier d'une fortune de parvenus. Fils du gentilhomme Pierre You de la Découverte, ce François d'Youville avait passé deux ans au Séminaire de Québec et y avait pris de bonnes manières, parmi les jeunes gens de la haute société de ce temps. Hélas! il fut un mari volage, noceur, infidèle et dur. L'épouse apprit aux lumières de la croix le dépassement de l'amour. Elle n'avait que 30 ans à la mort de Monsieur d'Youville, qui la laissait mère de deux enfants et ruinée. Femme remarquable et grande dame dans le plein sens du mot, elle chercha dans la prière, la lumière et le courage, s'employa à régler les fâcheuses dettes de son mari et à éduquer chrétiennement ses deux fils qui devinrent prêtres. La Providence la conduisit vers l'œuvre de l'Hôpital Général que les Sulpiciens lui proposèrent de relever; ce qu'elle fit à travers mille difficultés et épreuves. Cette femme intrépide au cœur magnifique, aux exceptionnels talents de réalisatrice, prit en charge toute la misère de Ville-Marie. Comptant sur la toute-puissante et bonne Providence du Père des cieux, et entourée de quelques compagnes qui s'engagèrent avec elle dans la profession religieuse, elle assuma cette tâche d'envergure pour l'amour des pauvres. C'est elle qu'on a appelée depuis la magicienne des gens mal pris, la grande Dame des pauvres, la mère de la charité universelle. Les vrais historiens, qui ont l'habitude d'exhumer les vieux documents, en apprendraient long sur cette histoire, en lisant la correspondance des grands du clergé et de la cour de France, des intendants et des gouverneurs du pays. Ils verraient, en ouvrant l'Encyclopédie du Canada, une annotation chaleureuse sur la MaisonMère des Sœurs Grises de Montréal. Il y est écrit : “C'est la maison de la charité; c'est la maison de l'amour, c'est la maison secourable érigée au centre du Canada.»

Et c'est de cette maison que partit Mère Mallet, le siècle d'après. La charité, comme l'évangile, est une vie. Il lui faut le mouvement, la poussée, la croissance. La communauté des Sœurs Grises déborda de Montréal, d'abord à Saint-Hyacinthe et à Bytown et puis à Québec en 1849. Elles y vinrent porter la compassion et l'humble service. La noble et sympathique ville de Québec et son peuple généreux étaient cette annéelà plongés dans la misère et le deuil. Dévastée en 1845, par deux conflagrations qui avaient anéanti les faubourgs Saint-Roch et Saint-JeanBaptiste, la ville se relevait péniblement de ces ruines, lorsque la population fut éprouvée par le fléau terrible des épidémies de typhus et de choléra. On avait fait bon accueil aux malheureux Irlandais émigrés chez nous. Des milliers étaient morts en mer et les autres sont porteurs de ce mal qui fera tant de victimes. Comme à Ville-Marie, depuis cent ans, les Seurs Grises furent appelées au secours de la souffrance de tous.

Marcelle Mallet était née en 1805, d'une humble famille qui habitait la Côte-des-Neiges à Montréal. Orpheline de père à 4 ans, et sa mère 
n'ayant pas ou peu de biens, la petite fille fut confiée, avec son jeune frère, à des parents adoptifs, un oncle et une tante, qui l'aimèrent comme leur propre enfant. Ils assurèrent son éducation et le bonheur de ses jeunes années, tout en caressant pour leur nièce d'avantageuses possibilités d'avenir. Quand un jour, celle-ci prit conscience de l'appel à la vie religieuse, elle eut peine à faire accepter son projet; mais d'une volonté forte comme l'acier, tout en refoulant ses larmes, elle dénoua les liens qui la retenaient. L'enfant de seize ans qui, pour le plus grand Amour, brise sans hésiter des obstacles qu'elle aime, n'annonce-t-elle pas une ascension très belle dans la voie du sacrifice? En 1821, il n'y avait sur l'île de Montréal que trois communautés de femmes. Écolière, Marcelle Mallet avait passé deux ans à la Congrégation de Notre-Dame qu'elle avait admirée; elle connaissait les Hospitalières de Saint-Joseph qu'elle trouvait remplies de charité pour les malades. Mais son cœur d'orpheline s'ouvrit à l'enfant sans famille et au pauvre sans foyer que protégeaient les Sours Grises.

La jeune religieuse apprit le grand savoir, c'est-à-dire l'art d'aimer Dieu, et le prochain. Cet enseignement lui fut donné par de bons guides et surtout par la Maîtresse des novices, religieuse d'un rare mérite et d'une haute vertu, Sour Catherine Boucher de Montbrun, dont le père, Étienne Boucher de Montbrun, était le petit-fils de Pierre Boucher de Boucherville. Dans la science des livres, Sœur Mallet n'est pas des plus brillantes. Au temps de ses études, les programmes ne visaient pas à préparer des bachelières; mais chez elle ces lacunes sont comblées par de nombreuses lectures et une très heureuse mémoire. Puis elle possède un bon et solide jugement; pas tellement de fantaisie, mais une finesse d'esprit et une affabilité toute naturelle et simple qui la rendait sympathique. Un constant sourire atténuait, en les éclairant de bonté, les traits plutôt sans délicatesse de son visage qui n'était pas joli, joli. Au moral, une rare facilité à laisser passer tout le monde pour s'écarter elle-même dans l'ombre; une calme fermeté, un esprit positif et pratique et les fortes vertus d'un caractère apte au renoncement et au dévouement sans défaillance. Telle était Mère Mallet.

Toute donnée à ses humbles tâches auprès des pauvres et des malades de l'hôpital, puis quelques années dans les charges, elle dispense à tous sa bonté compréhensive et sa maternelle sollicitude. En recevant son obédience de fondatrice, Mère Mallet était donc toute préparée à voler au secours de la détresse, vers Québec, la cité indigente et douloureuse, vers Québec criant sa misère à la charité. Ici encore, tout ce monde qui souffre, qui a besoin de pain, de toit, d'amour et de courage ! 


\section{QUÉBEC EN DÉTRESSE}

Il y avait bien aux Glacis, depuis 1831, une société de bienfaisance, les Dames de Charité, qui prit plus tard le nom de Société des Dames catholiques de Québec; reconnue et autorisée par la Législature provinciale et autorisée à recueillir des fonds. En 1849, le dévouement de ces Dames, secondé par les secours en argent de généreux bienfaiteurs, réussit à maintenir encore un orphelinat qui abrite 25 orphelines et 250 fillettes externes recevant gratuitement l'instruction. Hélas ! cette maison a aussi été ravagée par l'incendie; les murs n'en sont pas encore complètement relevés et les enfants ont été temporairement dispersés dans des familles secourables, ou placés à la Providence de Madame Gamelin, institut nouvellement ouvert à Montréal. De plus, à ce moment, on y a recueilli des jeunes victimes du typhus. Les secours sont devenus nettement insuffisants et Mgr Signay, l'archevêque, lance un appel chez les Sœurs Grises. Son coadjuteur, Mgr Pierre-Flavien Turgeon, est un jeune évêque supérieurement doué; avec ce bel optimisme audacieux qui caractérise les vrais apôtres, il entreprit les démarches nécessaires pour doter Québec d'une nouvelle congrégation religieuse. Il mit, à conclure les engagements avec Mgr Bourget, l'évêque de Montréal, et avec les Sœurs Grises, toute son énergie et son éloquence et mena l'affaire rondement avec dignité, bonté et une droiture irrésistible.

On aurait beaucoup à dire sur les antécédents de cette fondation, sur la pauvreté à Québec, sur l'œuvre des Dames Charitables et sur la personnalité et le dévouement de Mgr Turgeon. Encore cette fois, je me permets d'en référer aux ouvrages copieusement documentés de Sœur Sainte-Blanche, notre meilleure historiographe communautaire : Une fondatrice et son auvre et La charité en marche. Les historiens que vous êtes et tous ceux qui cultivent un peu de littérature, seront ravis d'y voir tant de trouvailles du lointain passé et tant de belles pages sur les annales de la ville de Québec. Également une large correspondance des évêques concernés et de l'Hôpital Général.

Les Sœurs Grises ont donc élu la fondatrice de l'œuvre québecoise.

\section{ET VOICI LA CHARITÉ}

En cette aube du 22 août 1849, la première page de l'histoire du nouvel institut s'écrit au fil du Saint-Laurent. Très tôt au lever du jour, le vapeur de la ligne Montréal-Québec, qui amenait les fondatrices, était en vue de la capitale. De tous les clochers tombe sinistre le glas qui endeuille la ville. Au petit orphelinat les enfants sont terrassés; les Dames ne suffisent pas à la tâche. Les nouvelles venues ont mis pied à terre. Elles sont prêtes. A Mère Mallet, la supérieure, il semble urgent de courir au chevet de ces pauvres enfants; le poste périlleux lui revient 
de droit. Mais Monseigneur, craignant la contagion, contrarie ce zèle et conduit le groupe à la Congrégation de Notre-Dame, où il est accueilli bien fraternellement. Ce même jour, visite aux Ursulines, à l'Hôtel-Dieu et à l'Hôpital Général. Le soir, Mère Mallet ose une nouvelle tentative; elle est comme hantée par la pensée des petits malades, que brûle la fièvre et qui l'attendent. L'évêque prend l'avis des médecins et la consigne cède.

Les Sœurs sont à leur poste et elles y veillent d'une presence actıve de jour et de nuit. De plus, on sollicite leur service pour les malades et les pauvres de l'extérieur. Elles multiplient les courses et les visites à domicile, assistent les mourants, affrontant le danger. C'est un bien mince renfort pour les religieuses de l'Hôtel-Dieu où il y a affluence et urgence; mais l'évêque, les Dames de l'Orphelinat et les médecins peuvent respirer un peu. Mgr le coadjuteur se montra un père et les Jésuites furent pour la jeune communauté ce que Saint-Sulpice était pour celle de Montréal. Comment louer assez la collaboration fraternelle et dévouée des Religieux de Saint-Vincent-de-Paul et des Frères des Écoles chrétiennes? Dès son arrivée, Mère Mallet accueille deux jeunes filles. Mlles Séraphine et Célina Roy, qui commencent aussitôt leur apprentissage de Sours de la Charité; ce sont les gens de Québec qui ont donné ce nom tout de suite et avec autorité aux Sœurs Grises, pour varier avec Montréal, pensons-nous. Autour de la fondatrice, nous trouvons donc aux tout premiers jours : Sœur Pilon, Sœur Perrin, Sœur Thériault, Sour Clément, venues de Montréal avec une novice irlandaise, Sour Alice Dunn; les deux nouvelles aspirantes se joignent donc à elles. Agées de 20 et 19 ans, elles sont filles de Madame François-Xavier Roy, qui entra elle-même en religion et fonda les Sœurs du Bon-Pasteur de Québec, dont elle fut la première supérieure, sous le nom de Mère Marie-du-SacréCœur. Son histoire n'est pas banale, ni celle de ses filles, et nous aurons le plaisir d'en entendre parler par Sœur Thérèse Boucher, conférencière à ce congrès.

A l'automne de cette année 1849, l'épidémie marque un temps de régression. Et à la faveur de ce bienfaisant répit, les Sœurs ouvrent leurs classes à 250 fillettes, installées aux Glacis, dans d'incommodes locaux de fortune qui n'offrent pas le dernier cri du confort. En 1854, les commissaires d'écoles demandèrent des Sœurs institutrices, pour obliger la population de Près-de-Ville, à l'école du Cap Diamant; elles consentirent à enseigner à ces petites filles en majorité irlandaises, sans pour autant négliger la nombreuse famille de leurs pauvres. Et puis le typhus ayant récidivé en différentes périodes, elles durent reprendre leur service des malades et furent même appelées à l'Hôpital de la Marine où étaient soignés de nombreux matelots irlandais. " $\mathrm{A}$ la bonne heure ! cela nous convient ". déclare Mère Mallet pour faire tomber encore une 
fois les craintes de Monseigneur. La Soeur de la Charité ne doit pas redouter les postes périlleux et bénévoles, pour lesquels on trouve peu de concurrence. Plus tard, en une circonstance semblable, ce sont les prêtres et les élèves du Petit Séminaire qui sont dirigés au même endroit; on ne peut les garder dans leur institution ni les rendre à leurs familles. Avec les médecins, les Sœurs sont là, plus ou moins infirmières, mais servantes, consolatrices et priantes.

Leur maison, au Côteau Sainte-Geneviève, s'appelle l'Hospice. Pendant des années, l'adresse se résume en ce seul mot : l'Hospice, et c'est suffisant pour la poste. Les gens et le courrier se rendent en droite ligne chez les Sœurs de la Charité. C'est la maison du pauvre, on y va pour toute nécessité. Elles sont les Sœurs de tout le monde et la porte est ouverte. - « On ne demande pas à celui qui entre s'il a un nom, mais s'il a une douleur. - Mais vous ne savez pas qui je suis, comment je m'appelle. - Vous êtes mon frère. " Ce dialogue entre Jean Valjean et le vieux curé dans Les Misérables, elle l'a repris souvent, Mère Mallet, avec ses protégés, vieillards, infirmes, vagabonds... Elle avait à un rare degré l'intelligence du pauvre et les archives gardent mille secrets sur sa charité; la tradition aussi, plus éloquente que les archives, actualise sous d'autres formes, les gestes de la fondatrice. Cet Hospice vient d'être agrandi et l'inauguration de l'annexe doit avoir lieu, mais à la veille de cette fête un incendie s'y déclare et le réduit à des murs noircis et lézardés. C'est la croix de feu, le sceau divin à cette œuvre qui refleurira sur les ruines.

\section{VERS DE NOUVELLES CEUVRES}

Les flammes montaient très haut, les courages, plus haut que les flammes et la charité les dépassa. Justement, la Providence a déjà décrété l'ère des fondations. Ce fut, après plusieurs petites écoles dans la ville, des couvents de campagne, des pensionnats de jeunes filles, des orphelinats, des maisons pour les vieillards et les aliénés. Au total, durant les cinquante premières années, 35 postes ou résidences, des " missions ", comme on disait. Sur ce nombre, 19 sont exclusifs à l'œuvre scolaire; 8 offrent les classes ainsi que l'accueil et le soin aux orphelins et aux personnes âgées; 8 sont à la fois orphelinat et hospice. Québec et sa région; Lévis, la rive sud et le Bas Saint-Laurent, jusqu'à la lointaine Gaspésie, Bois-Francs, Beauce, Cantons de l'Est, la farouche Pointe-aux-Esquimaux, Saint-Joseph-du-Labrador, de nos jours, Havre Saint-Pierre, trois Homes d'orphelins et de vieillards aux États-Unis. Toutes ces ouvres laborieuses et difficiles, ces éloignements, ces installations, ces rudes sacrifices, c'était bien la Chine et l'Afrique pour l'institut encore jeune. Et si on considère que, moins de 25 ans après sa fondation, il accepta de prendre en charge l'Asile de Beauport avec 
les frais, les travaux, les difficultés, les risques et les effectifs que cette œuvre immense exigea alors et de tous temps. Rien que cette histoire de Saint-Michel-Archange, depuis 1893, et de sa filiale de Saint-Ferdinand, prendrait tout le temps alloué pour cette causerie.

Mais c'est la FIN. Seulement, tout comme au cinéma, ce mot ne peut clore le message, qui doit se prolonger dans le souvenir et la bienfaisante méditation du passé.

C'est trop rapidement présenter cette esquisse historique et je le regrette. Heureusement il reste encore des choses importantes et des choses difficiles à accomplir. C'est à quoi les Sœurs de la Charité entendent bien s'employer, en suivant l'actualité des adaptations, voire des réformes, et aussi en réponse aux signes des temps, comme le demandent les décrets de l'immortel Vatican II et dans le magnifique esprit universel de Gaudium et Spes.

Quelle tâche splendide pour les femmes en gris et pour nous tous, que celle d'accroître et d'intensifier le contenu éternel d'amour dans notre monde !

Liliane HÉRoux, s.c.q.

Archiviste,

Rédactrice à l'Histoire de l'Institut

des SS. de la Charité de Québec. 